\title{
A Periodic Collaboration and Coexistence Management Model with the Oscillation Effect for Complex Mega Infrastructure Project under the Risk of Infection
}

\author{
Na Zhao $\mathbb{D}^{1,2}$ and T. C. E. Cheng $\mathbb{D}^{3}$ \\ ${ }^{1}$ School of Finance, Harbin University of Commerce, Harbin, Heilongjiang 150001, China \\ ${ }^{2}$ School of Economic and Management, Harbin Institute of Technology, Harbin, Heilongjiang 150001, China \\ ${ }^{3}$ Faculty of Business, The Hong Kong Polytechnic University, Kowloon, Hong Kong
}

Correspondence should be addressed to Na Zhao; zhaona@hit.edu.cn and T. C. E. Cheng; edwin.cheng@polyu.edu.hk

Received 8 April 2018; Accepted 12 June 2018; Published 2 October 2018

Academic Editor: Changzhi Wu

Copyright () $2018 \mathrm{Na}$ Zhao and T. C. E. Cheng. This is an open access article distributed under the Creative Commons Attribution License, which permits unrestricted use, distribution, and reproduction in any medium, provided the original work is properly cited.

In view of the intergroup structural relationships involved with the complex mega infrastructure projects (MIP), we develop a periodic collaboration and coexistence model that can efficiently curb the risk of infection and ensure normal, orderly progress. We conduct a systematic analysis of the periodic collaboration and the coexistence process for mega infrastructure projects. The results suggest that when a complex major engineering project develops the risk of infection and one group keeps expanding, assimilation or disappearance of the other group may occur, impeding the normal project construction process. If a disruption occurs, it is difficult to resume construction, and substantial economic losses can result. Furthermore, the periodic collaboration and coexistence management model with the oscillation effect can effectively prevent the risk of problems from spreading among groups. By doing so, the model stabilizes the intergroup structural balance in a complex major engineering system. In summary, our model and analysis help, to some extent, to elucidate the critical mechanisms influencing the risk of infection in engineering collaborative management and explain the collaboration and coexistence rules for a complex mega infrastructure project that may risk spreading infection to other parts of the project. The research findings, while further verifying and supplementing relevant theories, also provide beneficial references for collaborative management practices.

\section{Introduction}

Major infrastructure construction engineering is a general term for government-oriented large-scale complex engineering projects with a vital influence on economic and social development [1]. Driven by growing market demand, deepening global cooperation, government support, and huge capital input, complex engineering systems have not only profoundly changed production and lifestyle but also brought new challenges and opportunities to the management of mega infrastructure projects [2]. Most scholars focus on how to reduce construction engineering costs, accelerate the progress of construction engineering projects, and guarantee smooth construction engineering $[3,4]$. In addition, attention has gradually been paid to the risk of infection via the "butterfly effect," and efforts are being made to find an innovative theoretical method to assist in managing complex engineering projects (the risk of infection refers to the risk incurred by personality, emotion, and awareness factors, such as counterproductive work behavior, antisocial behavior, altruistic behavior, and conflicts among groups or individuals, which can influence the overall engineering interests; the risk of infection can induce other risks in a complex major engineering system or even trigger risks with more expansive and continuous influences).

1.1. Complexity of Engineering Management. Research into the complexity of engineering management originates from the complexity of engineering itself. Along with greater international cooperation, the increases in engineering quantity and complexity present huge challenges to decision-makers 
to improve the effectiveness of their decisions [5]. In response to this dilemma, theoreticians have started studying the complexity of mega infrastructure projects and have proposed new and systematic scientific methods to address the related issues [6]. Academic and engineering circles have gradually realized that the previous engineering management theories cannot effectively address the challenges brought by the complexity of mega infrastructure projects. A pioneering scholar, Ottino [7] found that engineering management personnel should be able to comprehend and tackle this complexity but that the ability to do so relies on the examination of many complex phenomena not within the sphere of engineering projects. Sauser and Boardman [8] also noted that knowledge management, an aspect of engineering management, has failed to catch up with the increasing complexity and integration of projects.

Humans drive the operation of engineering management systems. Therefore, research into the operation rules of these systems cannot be separated from the analysis of individuals and groups participating in engineering management [9]. To this end, Du and El-Gafy [10] examined the influence of the interplay between organizational and human factors using agent-based modelling (ABM). As one of the critical factors influencing engineering management, agent behavior arises from the interplay among certain psychological processes, psychological status, and personality psychology and is also subject to external environmental factors, such as culture and society [11]. Thus far, the behaviors of engineering management agents have been mainly investigated at the group and individual levels. The major influencing factors can be grouped into three categories, namely, psychological, behavioral, and cultural (see Table 1).

1.2. Risk of Infection in Mega Infrastructure Projects. Research into the risk of infection dates back to the 19th century, when Thornton [17] described the concept of the "risk spillover effect." Since then, many scholars have applied the idea to different research fields, including finance, insurance, information networks, and biomedicine. These works have indirectly enriched the research into the risk of infection, making it an emerging interdisciplinary research field. Unfortunately, there has not yet been any specific research on infection risk management for mega infrastructure projects.

Almeida et al. [18] noted that managing risk in mega infrastructure projects has become an increasing concern to stakeholders. "Man," "technology," and "external environment" constitute three critical factors influencing major engineering risks. To start with, the agent behaviors of "man" are influenced by personality psychology, psychological processes, and psychological status. Human factors influencing engineering risks include personality, emotion, motive, and consciousness [19]. Second, technological factors also influence the risk factors. Katalin et al. [20] theorized that engineering risk management and decision-making rely, to a large extent, on the strategy adopted to reduce risks. Cheng and Ding [21] described how to coordinate complexity through the establishment of clear boundaries. Finally, among external environmental factors influencing the risk of infection in engineering projects, the realization of external collaboration rests on the interaction between relevant agents, and intergroup collaboration is considered an effective approach to improving efficiency [22]. Horst [23] argued that homogeneity can contribute to the stability of the correlation system, while differentiation is attributable to the risk of infection. The infection effect first affects a minority and then spreads to the whole system through the interconnected relationship chain.

\subsection{Collaborative Management of Complex Engineering} Projects. The engineering system for a major project is a complex system with multiple departments involved in contrast to a simple physical facility construction [24]. Successful collaborative management is mainly determined by the interactions between the organizations and other relevant agents [25]. Engineering collaborative management can be defined as a cross-organizational collaboration process integrating multiple resource elements. In engineering collaborative management, efficient cooperative relationships among different groups and methods that can promote engineering efficiency and innovation are of equal importance. Some scholars have developed methods to improve organizational performance through structured system analysis and interactive management [26]. Therefore, an essential approach to improving complex engineering management is to improve the efficiency of the intergroup collaborative management.

Understanding the intergroup interactive complexity during the engineering collaborative management process, especially research on collaborative management mechanisms, such as intergroup interface and boundaries, is important [27]. In recent years, a few scholars have made preliminary investigations. For example, Antoniadis et al. [27] deemed that the key to successful implementation of an organizational engineering project lies in establishing a favorable communication and coordination mechanism across functional departments and creating a cohesive partnership. Senescu et al. [28] deepened the understanding of the correlation between project complexity and communication through in-depth analysis, and his work laid the foundation for the development of communication tools for more effective project management. Pauget and Wald [29] observed, after studying the relationship among members of research projects, that cooperative relationships between different groups or different individuals can influence the organizational network stability of mega infrastructure projects.

In summary, with the risk of infection, collaborative management of complex mega infrastructure projects is a top priority for engineering management research. However, the existing research mainly focuses on expounding on the importance and correlation of engineering management research. Based on previous engineering collaborative management research findings [30], and on life cycle theory and oscillation and dynamic behavior, this study is a pioneering work that, for the first time, defines the risk of infection in engineering projects, carries out in-depth analytical research into group collaboration within a complex engineering system under the influence of the risk of infection, and provides valuable insights. 
TABLE 1: Factors influencing individual and group behaviors in mega infrastructure projects.

\begin{tabular}{lcc}
\hline & Individual [12-14] & Group [15, 16] \\
\hline Psychology & Personality, emotion, motive, and consciousness & Trust, cohesion, and commitment \\
Behavior & Authorization, leadership, organizational citizen behavior & (OCB), counterproductive work behavior, altruistic behavior, \\
and reciprocal behavior & $\begin{array}{c}\text { Transactive memory system, shared mental model, conflict, } \\
\text { decision-making behavior, cooperation behavior, and } \\
\text { learning behavior }\end{array}$ \\
Culture & Job satisfaction, motive, and leadership & Learning, trust, learning, and conflict
\end{tabular}

We make the following major research contributions in this study: We (1) observe the periodic characteristics and group member characteristics of complex engineering projects through experiments and define relevant concepts concerning the risk of infection in complex engineering projects, (2) establish a periodic collaboration and coexistence model with the vibration effect and conduct empirical analysis of the model based on the theory of oscillation and using mathematical derivation, (3) reveal that when a complex major engineering project develops the risk of infection and one group keeps expanding, it might result in assimilation or disappearance of the other group or impede the major engineering project from normal construction, after which it is difficult to resume production, causing immeasurable economic losses, and (4) ascertain the conditions for the outbreak of infection and observe that the periodic collaboration and coexistence management model with the oscillation effect can effectively avoid the spread of infection between groups and guarantee a stable structure between groups in a complex major engineering system.

\section{Hypotheses}

Based on the characteristics of complex engineering systems, combining the theories of dynamics, oscillation, and life cycle, and in an environment of impending risk of infection, we observe that the engineering personnel density in the initial period of a complex major engineering project is relatively low, and there are structurally balanced resources for allocation. The numbers of personnel in different types of groups grow steadily along with the progress of the project construction period, but all these groups are still developing in independent environments [31].

As the initial period gradually moves into the maturity period, the engineering personnel density rises and there are fewer structurally balanced resources for allocation. In this period, different groups within the complex major engineering system might compete for their interests and attempt to increase their influence to occupy more resources. Consequently, the intergroup personnel relative density changes. On-site data of mega infrastructure projects suggests that "natural, small teams" exist in most major engineering construction groups. The "teammates" are either from the same area, have worked together for years, or are relatives. If adverse language and behavior occur in a teammate or several teammates, the connection between the personnel might cause conflict. In addition, if a group carrying or possessing the risk of infection is in a dominant position, then the infection influencing forces will grow and eventually assimilate or eliminate personnel in the other groups, thus decreasing the latter's personnel density (see Figure 1).

When the risk of infection is spread to a subsidiary engineering project, a construction period, or a department, the risk cannot be immediately classified when effective segregation measures are adopted. Under this condition, highpressure strategies are often adopted, which can damage the interests of irrelevant groups or force them to suspend work or even leave. In addition, high-pressure strategies can destroy the structural balance between the groups within an organization. Due to structural defects or the imbalance of major engineering personnel, the engineering project might come to a halt partially or completely, different engineering links will have difficulty in integrating with one another, and production cannot be resumed, resulting in serious economic losses and public security threats.

Therefore, regarding the periodic characteristics of major engineering systems, as well as the occurrence of traditional risks, we adopt an intergroup collaboration and coexistence mechanism through the risk infection medium (major engineering personnel group). Assuming and building a more practical periodic collaboration and coexistence model with the oscillation effect, we attempt to demonstrate and expound upon the role of the model in preventing the risk of infection from spreading among the groups involved in complex mega infrastructure projects.

\section{Modelling and Demonstration}

The structural interaction of different groups in a complex engineering system is usually subject to environmental influences, both internally and externally. When the risk of infection occurs, or is about to occur, some personnel in the group associated with the risk are, in the traditional approach, separated from the rest or directly expelled. However, based on the above discussion and existing theories, we adopt the periodic collaboration and coexistence management model to not only avoid triggering the risk of infection but also free the nonaffected groups from the influence of the risk, ensuring the smooth progress of the engineering project. To this end, we build the basic model, denoted as Model (1), as follows:

$$
\begin{aligned}
& \dot{\beta}(t)=\beta(t)\left(y_{1}(t)-x_{11}(t) \beta(t)-x_{12}(t) \lambda(t)\right)-D_{1}(\beta(t)), \\
& \dot{\lambda}(t)=\lambda(t)\left(y_{2}(t)-x_{21}(t) \beta(t)-x_{22}(t) \lambda(t)\right)-D_{2}(\lambda(t)),
\end{aligned}
$$




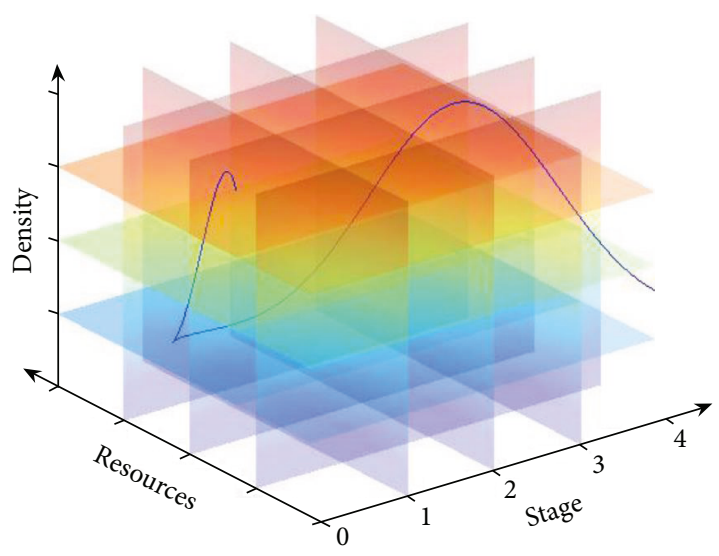

Figure 1: Changes in group personnel densities and resources in different periods of the life cycle.

where $D_{1}(\beta(t))$ denotes the influence of oscillatory collaborative management on the group $\beta(t)$ and $D_{2}(\lambda(t))$ denotes the influence of oscillatory collaborative management on the group $\lambda(t)$. Model (1) assumes that the collaborative management behavior is continuous. However, in reality, the occurrence of the risk of infection is periodic in major engineering management practices. This condition results in discreteness of infection risk prevention and control. In addition, considering the influence of the enrollment rates and turnover rates of different groups within the life cycle of mega infrastructure projects, as well as periodic changes, the traditional continuous, large-scale management methods to curb the risk of infection might be effectively replaced by collaborative management featuring discrete oscillation.

Based on the above argument, we develop the T-period collaboration and coexistence model with the oscillation effect as follows:

$$
\begin{array}{rlrl}
\dot{\beta}(t) & =\beta(t)\left(y_{1}(t)-x_{11}(t) \beta(t)-x_{12}(t) \lambda(t)\right), \quad & t \neq \tau k, k \in N, \\
\dot{\lambda}(t) & =\lambda(t)\left(y_{2}(t)-x_{21}(t) \beta(t)-x_{22}(t) \lambda(t)\right), \quad & t \neq \tau k, k \in N, \\
\beta\left(\tau_{k}^{+}\right) & =\left(1+h_{k}\right) \beta\left(\tau_{k}\right), \quad t \neq \tau_{k}, k \in N, \\
\lambda\left(\tau_{k}^{+}\right) & =\left(1+g_{k}\right) \lambda\left(\tau_{k}\right), \quad t \neq \tau_{k}, k \in N,
\end{array}
$$

where $y_{i}(t)$ and $x_{i j}(t)(i, j=1,2)$ constitute a continuous $T$-period function and meet the condition $x_{i j}(t) \geq 0(i, j=1$, $2, \quad i \neq j), x_{i i}>0$, and $N=\{1,2, \ldots\}$. Assume that $h_{k}$ and $g_{k}(k \in N)$ are constants, and there exists an integer $q>0$ such that $h_{k+q}=h_{k}, g_{k+q}=g_{k}$, and $\tau_{k+q}=\tau_{k}+T$. In view of real practice, there are natural restrictions that $1+h_{k}>0$ and $1+g_{k}>0$ if $k \in N$. $\tau$ denotes the period when oscillation collaboration is implemented. In other words, the moment of collaboration occurs at the discrete point $t=n \tau(n=0$, $1,2, \ldots)$; in an oscillating style, the $n$ time can be written as $t=n \tau^{+}$.

Model (2) attempts to prevent and control the risk of infection and considers the influence of collaborative management adopted by internal management personnel on the number of group members. In a complex major engineering system, there are different types of groups. In this study, we use Model (2) only to describe collaborative management between two group structures. $h_{k}$ and $g_{k}$, where $h_{k}<0$ and $g_{k}<0$, represent the oscillation collaboration degree between the engineering personnel group $\beta$ and the engineering personnel group $\lambda$, respectively.

From the life cycle perspective, the sustainable use of one resource (such as human resources) means the resource is never exhausted. In particular, the continuity of project construction usually indicates the resistance of a complex major engineering system to the risk of infection or an ability to quickly resume construction.

3.1. Continuous Existence of the System. Model $(2)$ at $(\beta, 0)$ must have a semitrivial periodic solution within the value $\bar{y}_{1}>(1 / T) \ln \left[\prod_{k=1}^{q}\left(1 / 1+h_{k}\right)\right]$, which can be simplified as $\left(\theta_{\left[y_{1}, x_{11}\right.}, 0\right)$. Similarly, $\operatorname{Model}(2)$ at $(0, \lambda)$ must have a semitrivial periodic solution within the value $\bar{y}_{2}>(1 / T) \ln \left[\prod_{k=1}^{q}(1 /\right.$ $\left.\left.1+g_{k}\right)\right]$, which can be simplified as $\left(0, \theta_{\left[y_{2}, x_{22}\right]}\right)$. Furthermore, the necessary and sufficient conditions can be expressed as $\theta_{y_{1}}:=\theta_{\left[y_{1}, x_{11}\right]}$ and $\theta_{y_{2}}:=\theta_{\left[y_{2}, x_{22}\right]}$, respectively. Through deduction, we obtain the following equations:

$$
\begin{aligned}
& \bar{y}_{1}=\frac{1}{T} \ln \left[\prod_{k=1}^{q} \frac{1}{1+h_{k}}\right]+\overline{x_{11} \theta_{y_{1}}}, \\
& \bar{y}_{2}=\frac{1}{T} \ln \left[\prod_{k=1}^{q} \frac{1}{1+g_{k}}\right]+\overline{x_{22} \theta_{y_{2}}} .
\end{aligned}
$$

In the following, we examine the situation when Model (2) does not allow coexistence whereby one side is fully assimilated or disappears. In other words, we seek to determine the conditions under which asymptotic steadiness of the trivial periodic solution $(0,0)$ and the semitrivial periodic solutions $\left(\theta_{y_{1}}, 0\right)$ and $\left(0, \theta_{y_{2}}\right)$ are achieved. We state and prove the following hypothesis.

\section{Hypothesis 1.}

(i) The trivial solution $(0,0)$ is linear and unsteady when Model (2) has a semitrivial periodic solution, that is, if and only if

$$
\bar{y}_{1}>\frac{1}{T} \ln \left[\prod_{k=1}^{q} \frac{1}{1+h_{k}}\right] \text { or } \bar{y}_{2}>\frac{1}{T} \ln \left[\prod_{k=1}^{q} \frac{1}{1+g_{k}}\right] .
$$

(ii) If $\bar{y}_{1}>(1 / T) \ln \left[\prod_{k=1}^{q}\left(1 / 1+h_{k}\right)\right]$, then $\left(\theta_{y_{1}}, 0\right)$ is linear and unsteady when $\bar{y}_{2}>(1 / T) \ln \left[\prod_{k=1}^{q}(1 / 1+\right.$ $\left.\left.g_{k}\right)\right]+\overline{x_{21} \theta_{y_{1}}}$ and is linearly steady when $\bar{y}_{2}<(1 / T)$ $\ln \left[\prod_{k=1}^{q}\left(1 / 1+g_{k}\right)\right]+\overline{x_{21} \theta_{y_{1}}}$.

(iii) If $\bar{y}_{2}>(1 / T) \ln \left[\prod_{k=1}^{q}\left(1 / 1+g_{k}\right)\right]$, then $\left(0, \theta_{y_{2}}\right)$ is linear and unsteady when $\bar{y}_{1}>(1 / T) \ln \left[\prod_{k=1}^{q}(1 / 1+\right.$ 


$$
\begin{aligned}
& \left.\left.h_{k}\right)\right]+\overline{x_{12} \theta_{y_{2}}} \text {, and is linearly steady when } \bar{y}_{1}<(1 / T) \\
& \ln \left[\prod_{k=1}^{q}\left(1 / 1+h_{k}\right)\right]+\overline{x_{12} \theta_{y_{2}}} .
\end{aligned}
$$

Proof 1 . The equation can be used to study the asymptotic steadiness of Model (2)'s periodic solution $(\bar{\beta}(t), \bar{\lambda}(t))$. After conversion by $(\beta(t), \lambda(t))=(\bar{\beta}(t)+a(t), \bar{\lambda}(t)+b(t))$, we have

$$
\left(\begin{array}{l}
a(t) \\
b(t)
\end{array}\right)=\Phi(t)\left(\begin{array}{l}
a(0) \\
b(0)
\end{array}\right),
$$

where $\Phi(t)$ meets the condition

$$
\frac{\mathrm{d} \Phi(t)}{\mathrm{d} t}=\left(\begin{array}{cc}
y_{1}-2 x_{11} \bar{\beta}-x_{12} \bar{\lambda} & -x_{12} \bar{\beta} \\
-x_{21} \bar{\lambda} & y_{2}-x_{21} \bar{\beta}-2 x_{22} \bar{\lambda}
\end{array}\right) \Phi(t)
$$

and $\Phi(0)=I$ is the unit matrix.

$$
\left(\begin{array}{l}
a\left(\tau_{k}^{+}\right) \\
b\left(\tau_{k}^{+}\right)
\end{array}\right)=\left(\begin{array}{cc}
1+h_{k} & 0 \\
0 & 1+g_{k}
\end{array}\right)\left(\begin{array}{l}
a\left(\tau_{k}\right) \\
b\left(\tau_{k}\right)
\end{array}\right),
$$

is given by the oscillation condition of Model (2).

Hence, if the module of the two eigenvalues of the following monodromy matrix

$$
M=\left(\begin{array}{cc}
\prod_{k=10}^{q}\left(1+h_{k}\right) & 0 \\
0 & \prod_{k=1}^{q}\left(1+g_{k}\right)
\end{array}\right) \Phi(T),
$$

is smaller than one, then the $T$-periodic solution is locally steady.

(i) The asymptotic steadiness of the trivial solution $(0,0)$.

The fundamental solution matrix of the trivial solution $(0,0)$ is

$$
\frac{d \Phi(t)}{\mathrm{d} t}=\left(\begin{array}{cc}
y_{1} & 0 \\
0 & y_{2}
\end{array}\right) \Phi(t)
$$

so its monodromy matrix is

$$
M=\left(\begin{array}{cc}
\prod_{k=1}^{q}\left(1+h_{k}\right) e^{\bar{y}_{1} T} & 0 \\
0 & \prod_{k=1}^{q}\left(1+t_{k}\right) e^{\bar{y}_{2} T}
\end{array}\right) .
$$

This proves that the trivial solution has two multipliers at the point $(0,0): \beta_{1}=\prod_{k=1}^{q}\left(1+h_{k}\right) e^{\bar{y}_{1} T}$ and $\beta_{2}=\prod_{k=1}^{q}\left(1+g_{k}\right)$ $e^{\bar{y}_{2} T}$. Therefore, the conclusion stated in (i) is correct.

(ii) The linear steadiness of the semitrivial periodic solution.

Corresponding to every semitrivial solution $\left(\theta_{y_{1}}, 0\right)$ is

$$
\frac{d \Phi(t)}{\mathrm{d} t}=\left(\begin{array}{cc}
y_{1}-2 x_{11} \theta_{y_{1}} & -2 x_{12} \theta_{y_{1}} \\
0 & y_{2}-x_{21} \theta_{y_{1}}
\end{array}\right) \Phi(t) .
$$

Therefore, the fundamental solution matrix of the semitrivial solution is as follows:

$$
\Phi(t)=\left(\begin{array}{cc}
\exp \int_{0}^{t}\left(y_{1}-2 x_{11} \theta_{y_{1}}\right) \mathrm{d} s & * \\
0 & \exp \int_{0}^{t}\left(y_{2}-2 x_{21} \theta_{y_{1}}\right) \mathrm{d} s
\end{array}\right) .
$$

Since the following computing process does not rely on $\left({ }^{*}\right)$, there is no need to provide its exact formula. The steadiness of the semitrivial solution $\left(\theta_{y_{1}}, 0\right)$ is confirmed by the two eigenvalues of the following monodromy matrix

$$
M=\left(\begin{array}{cc}
\prod_{k=1}^{q}\left(1+h_{k}\right) & 0 \\
0 & \prod_{k=1}^{q}\left(1+g_{k}\right)
\end{array}\right) \Phi(T) .
$$

where

$$
\begin{aligned}
\beta_{1} & =\prod_{k=1}^{q}\left(1+h_{k}\right) \exp \int_{0}^{T}\left(y_{1}-2 x_{11} \theta_{y_{1}}\right) \mathrm{d} s \\
& =\prod_{k=1}^{q}\left(1+h_{k}\right) \exp \left[\int_{0}^{T}\left(y_{1}-x_{11} \theta_{y_{1}}\right) \mathrm{d} s-\int_{0}^{T} x_{11} \theta_{y_{1}} \mathrm{~d} s\right] \\
& =\prod_{k=1}^{q}\left(1+h_{k}\right) \exp \left[\ln \prod_{k=1}^{q} \frac{1}{1+h_{k}}\right] \exp \left[-\int_{0}^{T} x_{11} \theta_{y_{1}} \mathrm{~d} s\right] \\
& =\exp \left[-\overline{x_{11} \theta_{y_{1}}} T\right]=\exp \left[-T\left(\bar{y}_{1}-\frac{1}{T} \ln \prod_{k=1}^{q} \frac{1}{1+h_{k}}\right)\right],
\end{aligned}
$$

and

$$
\begin{aligned}
\beta_{2} & =\prod_{k=1}^{q}\left(1+g_{k}\right) \exp \int_{0}^{T}\left(y_{2}-x_{21} \theta_{y_{1}}\right) \mathrm{d} s \\
& =\prod_{k=1}^{q}\left(1+g_{k}\right) \exp \left(\bar{y}_{2}-\overline{x_{21} \theta_{y_{1}}}\right) T .
\end{aligned}
$$

If $\bar{y}_{1}>(1 / T) \ln \left[\prod_{k=1}^{q}\left(1 / 1+h_{k}\right)\right]$, then $\beta_{1}<1$ and the linear steadiness of the semitrivial solution $\left(\theta_{y_{1}}, 0\right)$ is confirmed by $\bar{y}_{2}-(1 / T) \ln \left[\prod_{k=1}^{q}\left(1 / 1+g_{k}\right)\right]-\overline{x_{21} \theta_{y_{1}}}$. If $\bar{y}_{2}>(1 / T) \ln [$ $\left.\prod_{k=1}^{q}\left(1 / 1+g_{k}\right)\right]+\overline{x_{21} \theta_{y_{1}}}$, then $\beta_{2}>1$ and the unsteady manifold of the semitrivial solution $\left(\theta_{y_{1}}, 0\right)$ is one-dimensional. If $\bar{y}_{2}=(1 / T) \ln \left[\prod_{k=1}^{q}\left(1 / 1+g_{k}\right)\right]+\overline{x_{21} \theta_{y_{1}}}$, then $\left(\theta_{y_{1}}, 0\right)$ features neutral steadiness. If $\bar{y}_{2}<(1 / T) \ln \left[\prod_{k=1}^{q}\left(1 / 1+g_{k}\right)\right]+\overline{x_{21} \theta_{y_{1}}}$, then $\beta_{2}<1$ and $\left(\theta_{y_{1}}, 0\right)$ is linearly steady. 
(iii) The linear steadiness of the semitrivial periodic solution.

Similarly, the linear steadiness of the semitrivial periodic solution $\left(0, \theta_{y_{2}}\right)$ is confirmed by $\bar{y}_{1}-(1 / T) \ln \left[\prod_{k=1}^{q}(1 / 1+\right.$ $\left.\left.h_{k}\right)\right]+\overline{x_{12} \theta_{y_{2}}}$.

The steadiness of the semitrivial periodic solutions $\left(\theta_{y_{1}}\right.$, $0)$ and $\left(0, \theta_{y_{2}}\right)$ suggests that when the risk of infection occurs, one personnel group in a major engineering system might be fully assimilated or disappear and the other personnel group might expand. Therefore, to guarantee equilibrium steadiness of the groups in a complex major engineering system, a necessary condition for coexistence of the two kinds of personnel is that the following two equations exist. If one group is fully infected and thus becomes fully assimilated or disappears, the engineering construction might be unable to carry on normally and it is difficult to resume construction, resulting in tremendous economic losses. In other words, all the trivial periodic solutions and semitrivial periodic solutions are linearly unsteady, that is,

$$
\begin{aligned}
& \bar{y}_{1}>\frac{1}{T} \ln \left[\prod_{k=1}^{q} \frac{1}{1+h_{k}}\right]+\overline{x_{12} \theta_{y_{2}}}, \\
& \bar{y}_{2}>\frac{1}{T} \ln \left[\prod_{k=1}^{q} \frac{1}{1+g_{k}}\right]+\overline{x_{21} \theta_{y_{1}}} .
\end{aligned}
$$

In the following section, we examine how to achieve sustainable coexistence and avoid the occurrence of the risk of infection when the personnel groups are structurally stable in the major engineering system. We write Model (2) under the condition of coexistence as follows:

Assume that there exist a constant $m(M>0)$ and a finite time $T_{0}$, which are independent of the system's initiative values, and make all the initiative values of Model (2) as the solution $a_{i}(t)$ of $a_{i}\left(0^{+}\right)>0$. When $t>T_{0}$, then $m<a_{i}(t)<$ $M(i=1,2)$ and Model (2) is coexisting. Here, $T_{0}$ depends on the initial value $a_{i}\left(0^{+}\right)$.

Hypothesis 2. If the following inequalities are valid,

$$
\begin{aligned}
& \bar{y}_{1}>\frac{1}{T} \ln \left[\prod_{k=1}^{q} \frac{1}{1+h_{k}}\right]+\overline{x_{12} \theta_{y_{2}}}, \\
& \bar{y}_{2}>\frac{1}{T} \ln \left[\prod_{k=1}^{q} \frac{1}{1+g_{k}}\right]+\overline{x_{21} \theta_{y_{1}}},
\end{aligned}
$$

then Model (2) suggests that the two kinds of engineering personnel groups coexist and collaborate with each other in a complex major engineering system. Based on Model (2), when $t$ is sufficiently large, we have

$$
\begin{aligned}
\dot{\beta}(t) & \leq \beta(t)\left(y_{1}(t)-x_{11}(t) \beta(t)\right), \quad t \neq \tau_{k}, k \in N, \\
\beta\left(\tau_{k}^{+}\right) & =\left(1+h_{k}\right) \beta\left(\tau_{k}\right), \quad t=\tau_{k}, k \in N,
\end{aligned}
$$

where $\beta(t) \leq \theta_{y_{1}}$. Similarly, when $t$ is sufficiently large, we have $\lambda(t) \leq \theta_{y_{2}}$. Then, there exists a constant $T_{1}>0$ such that when $t \geq T_{1}$, then $\beta(t) \leq \theta_{y_{1}}$ and $\lambda(t) \leq \theta_{y_{2}}$. Thus, when $t>$ $T_{1}$, we have

$$
\begin{aligned}
& \dot{\beta}(t) \geq \beta(t)\left(y_{1}(t)-x_{12}(t) \theta_{y_{2}}-x_{11}(t) \beta(t)\right), \quad t \neq \tau_{k}, k \in Z^{+}, \\
& \beta\left(\tau_{k}^{+}\right)=\left(1+h_{k}\right) \beta\left(\tau_{k}\right), \quad t=\tau_{k}, k \in Z^{+},
\end{aligned}
$$

and, when $t$ is sufficiently large, we have $\beta(t) \geq \theta_{\left[y_{1}-x_{12} \theta_{y_{2}}, x_{11}\right]}$ $>0$. Similarly, when $t$ is sufficiently large, we have $\lambda(t) \geq$ $\theta_{\left[y_{2}-x_{21} \theta_{y_{1}}, x_{22}\right]}>0$. Hence, there exists a constant $\bar{T}>T_{1}$ such that when $t \geq \bar{T}$, then $\beta(t) \geq \theta_{\left[y_{1}-x_{12} \theta_{y_{2}}, x_{11}\right]}$ and $\lambda(t) \geq$ $\theta_{\left[y_{2}-x_{21} \theta_{y_{1}}, x_{22}\right]}$. Assume that

$\rho=\inf \left\{\theta_{\left[y_{1}-x_{12} \theta_{y_{2}}, x_{11}\right]}(t), \quad \theta_{\left[y_{2}-x_{21} \theta_{y_{1}}, x_{22}\right]}(t): t \in[0, T]\right\}, \quad 0<\rho<\infty$, $\sigma=\sup \left\{\theta_{y_{1}}(t), \quad \theta_{y_{2}}(t) \in[0, T]\right\}, \quad \rho \leq \sigma<\infty$.

Then, when $t \geq \bar{T}$, we have $\rho \leq \beta(t) \leq \sigma$ and $\rho \leq \lambda(t) \leq \sigma$.

3.2. Bifurcation of the Solution. In this study, we use bifurcation theory to discuss the bifurcation of Model (2) near the semitrivial periodic solutions $\left(\theta_{y_{1}}, 0\right)$ and $\left(0, \theta_{y_{2}}\right)$. To validate the analytical findings of this study, first examine the two results of the linear oscillation periodic equation.

Hypothesis 3. Assume that $c_{i j} \in P C_{T}$.

(i) If $\overline{c_{11}} \neq(1 / T) \ln \left[\prod_{k=1}^{q}\left(1 / 1+c_{k}\right)\right]$ and $\overline{c_{22}} \neq(1 / T) \ln$ $\left[\prod_{k=1}^{q}\left(1 / 1+d_{k}\right)\right]$, then the periodic linear homogeneous oscillation equation can be written as follows:

$$
\begin{aligned}
\dot{b}_{1}(t) & =c_{11} b_{1}(t)+c_{12} b_{2}(t), \quad t \neq \tau_{k}, k \in N, \\
\dot{b}_{2}(t) & =c_{22} b_{2}(t), \quad t \neq \tau_{k}, k \in N, \\
b_{1}\left(\tau_{k}^{+}\right) & =\left(1+c_{k}\right) b_{1}\left(\tau_{k}\right), \quad t=\tau_{k}, k \in N, \\
b_{2}\left(\tau_{k}^{+}\right) & =\left(1+d_{k}\right) b_{2}\left(\tau_{k}\right), \quad t=\tau_{k}, k \in N .
\end{aligned}
$$

On $P C_{T} \times P C_{T}$, there is no nontrivial periodic solution. Under this condition, if $\left(f_{1}, f_{2}\right) \in P C_{T} \times P C_{T}$, we write the nonhomogeneous oscillation equation as follows:

$$
\begin{aligned}
\dot{z}_{1}(t) & =c_{11} z_{1}(t)+c_{12} z_{2}(t)+f_{1}, \quad t \neq \tau_{k}, k \in N, \\
\dot{z}_{2}(t) & =c_{22} z_{2}(t)+f_{2}, \quad t \neq \tau_{k}, k \in N, \\
z_{1}\left(\tau_{k}^{+}\right) & =\left(1+c_{k}\right) z_{1}\left(\tau_{k}\right), \quad t=\tau_{k}, k \in N, \\
z_{2}\left(\tau_{k}^{+}\right) & =\left(1+d_{k}\right) z_{2}\left(\tau_{k}\right), \quad t=\tau_{k}, k \in N .
\end{aligned}
$$

The $T$-periodic solution is exclusive and $\left(z_{1}, z_{2}\right) \in P C_{T} \times$ $P C_{T}$. Furthermore, the operator $L: P C_{T} \times P C_{T} \rightarrow P C_{T} \times$ $P C_{T}$, defined by $\left(z_{1}, z_{2}\right)=L\left(f_{1}, f_{2}\right)$, is linear and relatively compact. 
(ii) If $\overline{c_{22}}=(1 / T) \ln \left[\prod_{k=1}^{q}\left(1 / 1+d_{k}\right)\right]$ and $\overline{c_{11}} \neq(1 / T) \ln$ $\left[\prod_{k=1}^{q}\left(1 / 1+c_{k}\right)\right]$ are valid, then (22) has only one $T$-periodic solution on $P C_{T} \times P C_{T}$.

Proof 1.

(i) Since

$$
b_{2}(t)=\prod_{0 \leq \tau_{k}<t}\left(1+d_{k}\right) b_{2}(0) \exp \int_{0}^{T} c_{22}(s) \mathrm{d} s,
$$

$\overline{c_{22}} \neq(1 / T) \ln \left[\prod_{k=1}^{q}\left(1 / 1+d_{k}\right)\right] \quad$ suggests $\quad b_{2} \notin P C_{T}$ unless $b_{2}=0$. Similarly, since

$$
b_{1}(t)=\prod_{0 \leq \tau_{k}<t}\left(1+c_{k}\right) b_{1}(0) \exp \int_{0}^{T} c_{11}(s) \mathrm{d} s,
$$

$\overline{c_{11}} \neq(1 / T) \ln \left[\prod_{k=1}^{q}\left(1 / 1+c_{k}\right)\right] \quad$ suggests $\quad b \notin P C_{T}$ unless $b_{1}=0$. Under the condition

$$
\begin{gathered}
\dot{z}_{2}(t)=c_{22} z_{2}(t)+f_{2}, \quad t \neq \tau_{k}, k \in N, \\
z_{2}\left(\tau_{k}^{+}\right)=\left(1+d_{k}\right) z_{2}\left(\tau_{k}\right), \quad t=\tau_{k}, k \in N,
\end{gathered}
$$

there is only one $T$-periodic solution, and $L_{2}: P C_{T}$ $\rightarrow P C_{T}$ is defined by $z_{2}=L_{2} f_{2}$ and is linear and relatively compact. Furthermore, when $f_{3}=c_{12} L_{2} f_{2}+f_{1}$ $\in P C_{T}$, the equation

$$
\begin{aligned}
\dot{z}_{1}(t) & =c_{11} z_{1}(t)+f_{3}, \quad t \neq \tau_{k}, k \in N, \\
z_{1}\left(\tau_{k}^{+}\right) & =\left(1+c_{k}\right) z_{2}\left(\tau_{k}\right), \quad t=\tau_{k}, k \in N,
\end{aligned}
$$

has only one $T$-periodic solution, and $z_{1}=L_{1} f_{3}$ defines a linear and relatively compact operator $L_{1}$ : $P C_{T} \rightarrow P C_{T}$. Therefore, (22) has only one $T$-periodic solution determined by $\left(z_{1}, z_{2}\right)=L\left(f_{1}, f_{2}\right)$ and is the only solution on $P C_{T} \times P C_{T}$, where

$$
L\left(f_{1}, f_{2}\right)=\left(L_{1}\left(c_{12} L_{2} f_{2}+f_{1}\right), L_{2} f_{2}\right)
$$

(ii) Under the hypothesis, the solution adopting a random $b_{2}(0)$ as the initial value is a $T$-periodic solution and is given by (23). If $\overline{c_{11}} \neq(1 / T)\left[\prod_{k=1}^{q}\left(1 / 1+c_{k}\right)\right]$, then $\dot{b}(t)=c_{11} b(t)$ does not have a nontrivial periodic solution on $P C_{T}$. Then, we have

$$
\begin{gathered}
\dot{b}_{1}(t)=c_{11} b_{1}(t)+c_{12} \prod_{0 \leq \tau_{k}<t}\left(1+d_{k}\right) b_{2}(0) \\
\cdot \exp \int_{0}^{t} c_{22}(s) \mathrm{d} s, \quad t \neq \tau_{k}, \\
b_{1}\left(\tau_{k}^{+}\right)=\left(1+c_{k}\right) b_{1}\left(\tau_{k}\right), \quad t=\tau_{k}, k \in N .
\end{gathered}
$$

On $P C_{T}$, the $T$-periodic solution is exclusive. The hypothesis is true.

Hypothesis 4. Assume that $x \in P C_{T}$ and $\bar{x}=(1 / T)\left[\prod_{k=1}^{q}(1 / 1+\right.$ $\left.c_{k}\right)$ ], then

$$
\begin{aligned}
\dot{a} & =x a+f, \quad t \neq \tau_{k}, k \in N, \\
a\left(\tau_{k}^{+}\right) & =\left(1+c_{k}\right) a\left(\tau_{k}\right), \quad t=\tau_{k}, k \in N .
\end{aligned}
$$

The necessary and sufficient condition for the $T$-periodic solution $x \in P C_{T}$ is as follows:

$$
\int_{0}^{T} \prod_{0 \leq \tau_{k}<t} \frac{1}{1+c_{k}} \exp \left[-\int_{0}^{t} x(\beta) \mathrm{d} \beta\right] f d t=0 .
$$

Proof 1 . The matrix of the linear homogeneous oscillation equation is as follows:

$$
\begin{aligned}
\dot{a} & =a x, \quad t \neq \tau_{k}, k \in N, \\
a\left(\tau_{k}^{+}\right) & =\left(1+c_{k}\right) a\left(\tau_{k}\right), \quad t=\tau_{k}, k \in N .
\end{aligned}
$$

The matrix is $A(t, s)=\prod_{s \leq \tau_{k}<t}\left(1+c_{k}\right) \exp \int_{s}^{t} x(\beta) \mathrm{d} \beta$, $\infty<s \leq t<\infty$. Then, (31) is the exclusive multiplier

$$
\mu=A\left(T, 0^{+}\right)=\prod_{k=1}\left(1+c_{k}\right) \exp \int_{0}^{T} x(s) \mathrm{d} s=1 .
$$

Hence, all the solutions of (31) are T-periodic solutions and all the solutions of the adjoining equation

$$
\begin{gathered}
\dot{b}=-x b, \quad t \neq \tau_{k}, k \in N, \\
b\left(\tau_{k}^{+}\right)=\frac{1}{1+c_{k}} b\left(\tau_{k}\right), \quad t=\tau_{k}, k \in N,
\end{gathered}
$$

are also $T$-periodic. In particular, $b=\psi(t)=\prod_{0 \leq \tau_{k}<t}(1 / 1+$ $\left.c_{k}\right) \exp \left[-\int_{0}^{t} x(\beta) \mathrm{d} \beta\right]$ is also a $T$-periodic solution of (27), where $\psi\left(T^{+}\right)=\psi\left(0^{+}\right)=1$. The necessary and sufficient condition for (29) to have only one $T$-periodic solution is $\int_{0}^{T} \psi(t) f d t=0$, that is,

$$
\int_{0}^{T} \prod_{0 \leq \tau_{k}<t} \frac{1}{1+c_{k}} \exp \left[-\int_{0}^{t} x(\beta) \mathrm{d} \beta\right] f d t=0 .
$$

Assume $\bar{y}_{1}>(1 / T) \ln \left[\prod_{k=1}^{q}\left(1 / 1+h_{k}\right)\right]$ and regard $y_{2}$ as the bifurcation referential value. This condition suggests that $\xi_{*}=(1 / T) \ln \left[\prod_{k=1}^{q}\left(1 / 1+g_{k}\right)\right]+\overline{x_{21} \theta_{y_{1}}}$ is the bifurcation value of the positive periodic solution bifurcated from the semitrivial periodic solution $\left(\theta_{y_{1}}, 0\right)$. Assume that $y_{2}(t)=$ $\xi+p_{2}(t)$, where $\bar{p}_{2}=0$. Then,

$$
a_{21}(t)=\prod_{0 \leq \tau_{k}<t}\left(1+g_{k}\right) \exp \left[\int_{0}^{t}\left(p_{2}+\xi_{*}-x_{21} \theta_{y_{1}}\right) \mathrm{d} s\right] \in P C_{T} .
$$

Assume $a_{11}$ to be the only $T$-periodic solution of the following linear oscillation differential equation: 


$$
\begin{aligned}
\dot{a}_{11}(t) & =\left(y_{1}-2 x_{11} \theta_{y_{1}}\right) a_{11}(t)-x_{12}(t) \theta_{y_{1}} a_{21}(t), \quad t \neq \tau_{k}, k \in N, \\
a_{11}\left(\tau_{k}^{+}\right) & =\left(1+h_{k}\right) a_{11}\left(\tau_{k}\right), \quad t=\tau_{k}, k \in N .
\end{aligned}
$$

Then

$$
a_{11}(t)=-\int_{0}^{t} G(t, s) x_{12} \theta_{y_{1}} a_{21}(s) \mathrm{d} s,
$$

where

$$
\begin{aligned}
& G(t, s) \\
& =\left\{\begin{array}{l}
A(t)(1-A(t))^{-1} A^{-1}(s), \quad(0<s<t \leq T), \\
A(t+T)(1-A(T))^{-1} A(-1)(s), \quad(0<t \leq s \leq T), \\
G(t-k T, s-j T), \quad(k T<t \leq k T+T, j T<s \leq j T+T, k \in N, j \in N),
\end{array}\right.
\end{aligned}
$$

and $A(t)=\prod_{0 \leq \tau_{k}<t}\left(t+h_{k}\right) \exp \int_{0}^{t}\left(y_{2}-2 x_{11} \theta_{y_{1}}\right) \mathrm{d} s$.

Note that $a_{21}(t)>0$ exists if $t>0$. Since

$$
\begin{aligned}
A(t)= & \prod_{k=1}^{q}\left(1+h_{k}\right) \exp \left[\int_{0}^{T}\left(y_{1}-2 x_{11} \theta_{y_{1}}\right) \mathrm{d} s\right] \\
= & \prod_{k=1}^{q}\left(1+h_{k}\right) \exp \left[\int_{0}^{T}\left(y_{1}-x_{11} \theta_{y_{1}}\right) \mathrm{d} s\right] \exp \\
& \cdot\left[\int_{0}^{T}\left(-x_{11} \theta_{y_{1}}\right) \mathrm{d} s\right]=\exp \left(-\int_{0}^{T} x_{11} \theta_{y_{1}} \mathrm{~d} s\right)<1, \quad G(t, s)>0 .
\end{aligned}
$$

Then, for any $t, a_{11}(t)<0$.

Define $\xi_{1}=\overline{x_{21} a_{11}(t)+x_{22}(t) a_{21}(t)}$. The main conclusions are stated in the following hypothesis.

Hypothesis 5. Assume $y_{i}(t) . x_{i j} \in P C_{T}(i, j=1,2)$ is valid in terms of $\bar{y}_{1}>(1 / T) \ln \left[\prod_{k=1}^{q}\left(1 / 1+h_{k}\right)\right]$ and $x_{21} \theta_{y_{1}}>(1 / T)$ $\ln \left[\prod_{k=1}^{q}\left(1+g_{k}\right)\right]$. For any $t>0$, there is $x_{i j}(t)>0$. Then,

(i) If $\xi_{1}>0$, then there exists a constant $c_{0}>0$, which is sufficiently small to ensure that, in terms of every $y_{2}(t)\left(y_{2}(t) \in P C_{T}\right)$ existing under the restriction of $\xi_{*}<\bar{y}_{2}<\xi_{*}+c_{0}$, Model (2) has a periodic solution $(\beta, \lambda) \in P C_{T} \times P C_{T}$, which can guarantee $\beta_{t}<\theta_{y_{1}}$ and $\lambda(t)>0$ as long as $t>0$.

(ii) If $\xi_{1}<0$, there exists a constant $d_{0}>0$, which is sufficiently small to ensure that, in terms of every $y_{2}(t)\left(y_{2}(t) \in P C_{T}\right)$ existing under the restriction of $\xi_{*}-d_{0}<\bar{y}_{2}<\xi_{*}$, Model (2) has a periodic solution $(\beta, \lambda) \in P C_{T} \times P C_{T}$, which can guarantee $\beta(t)<\theta_{y_{1}}$ and $\lambda(t)>0$ as long as $t>0$.

Proof 1. In Model (2), after conversion, we have $a_{1}=\beta(t)-$ $\theta_{y_{1}} a_{2}=\lambda(t)$. Then,

$$
\begin{aligned}
\dot{a}_{1}(t) & =\left(y_{1}-2 x_{11} \theta_{y_{1}}\right) a_{1}-x_{12} \theta_{y_{1}} a_{2}+g_{1}\left(a_{1}, a_{2}\right), \quad t \neq \tau_{k}, k \in N, \\
\dot{a}_{2}(t) & =\left(y_{2}-x_{21} \theta_{y_{1}}\right) a_{2}+g_{2}\left(a_{1}, a_{2}\right), \quad t \neq \tau_{k}, k \in N, \\
a_{1}\left(\tau_{k}^{+}\right) & =\left(1+h_{k}\right) a_{1}\left(\tau_{k}\right), \quad t=\tau_{k}, k \in N, \\
a_{2}\left(\tau_{k}^{+}\right) & =\left(1+g_{k}\right) a_{2}\left(\tau_{k}\right), \quad t=\tau_{k}, k \in N,
\end{aligned}
$$

where $g_{1}\left(a_{1}, a_{2}\right)=-x_{11} a_{1}^{2}-x_{12} a_{1} a_{2}$ and $g_{2}\left(a_{1}, a_{2}\right)=-x_{21} a_{1}$ $a_{2}-x_{22} a_{2}^{2}$.

Define $p_{2}(t)=y_{2}(t)-\xi$, where $\xi=\bar{y}_{2}$. Then, $y_{2}=p_{2}+\xi$ and $\bar{p}_{2}=0$. Then, we write (40) as follows:

$$
\begin{aligned}
\dot{a}_{1}(t) & =\left(y_{1}-2 x_{11} \theta_{y_{1}}\right) a_{1}-x_{12} \theta_{y_{1}} a_{2}+g_{1}\left(a_{1}, a_{2}\right), \quad t \neq \tau_{k}, k \in N, \\
\dot{a}_{2}(t) & =\left(p_{2}-x_{21} \theta_{y_{1}}\right) a_{2}+\xi a_{2}+g_{2}\left(a_{1}, a_{2}\right), \quad t \neq \tau_{k}, k \in N, \\
a_{1}\left(\tau_{k}^{+}\right) & =\left(1+h_{k}\right) a_{1}\left(\tau_{k}\right), \quad t=\tau_{k}, k \in N, \\
a_{2}\left(\tau_{k}^{+}\right) & =\left(1+g_{k}\right) a_{2}\left(\tau_{k}\right), \quad t=\tau_{k}, k \in N .
\end{aligned}
$$

Its linear homogeneous function can be written as follows:

$$
\begin{aligned}
\dot{\delta}_{1}(t) & =\left(y_{1}-2 x_{11} \theta_{y_{1}}\right) \delta_{1}-x_{12} \theta_{y_{1}} \delta_{2}, \quad t \neq \tau_{k}, k \in N, \\
\dot{\delta}_{2}(t) & =\left(p_{2}-x_{21} \theta_{y_{1}}\right) \delta_{2}, \quad t \neq \tau_{k}, k \in N, \\
\delta_{1}\left(\tau_{k}^{+}\right) & =\left(1+h_{k}\right) \delta_{1}\left(\tau_{k}\right), \quad t=\tau_{k}, k \in N, \\
\delta_{2}\left(\tau_{k}^{+}\right) & =\left(1+g_{k}\right) \delta_{2}\left(\tau_{k}\right), \quad t=\tau_{k}, k \in N .
\end{aligned}
$$

Noting that $\overline{x_{21} \theta_{y_{1}}}>(1 / T) \ln \left[\prod_{k=1}^{q}\left(1+g_{k}\right)\right]$, we have

$$
\overline{c_{22}}=\overline{p_{2}-x_{21} \theta_{y_{1}}}=-\overline{x_{21} \theta_{y_{1}}}<\frac{1}{T} \ln \left[\prod_{k=1}^{q} \frac{1}{1+g_{k}}\right],
$$

and

$$
\begin{aligned}
\overline{c_{11}} & =\overline{y_{1}-2 x_{11} \theta_{y_{1}}}=\overline{y_{1}-x_{11} \theta_{y_{1}}}-\overline{x_{11} \theta_{y_{1}}} \\
& =\frac{1}{T} \ln \left[\prod_{k=1}^{q} \frac{1}{1+h_{k}}\right]-\overline{x_{11} \theta_{y_{1}}} \neq \frac{1}{T} \ln \left[\prod_{k=1}^{q} \frac{1}{1+h_{k}}\right] .
\end{aligned}
$$

We apply (41) to obtain the relatively compact linear operator $L: P C_{T} \times P C_{T} \rightarrow P C_{T} \times P C_{T}$. Based on $L$, we write (41) as the following equivalent operator function

$$
\left(a_{1}, a_{2}\right)=\xi L^{*}\left(a_{1}, a_{2}\right)+G\left(a_{1}, a_{2}\right)
$$

where 


$$
L^{*}\left(a_{1}, a_{2}\right)=\left(L_{1}\left(-x_{12} \theta_{y_{1}} L_{2} a_{2}\right), L_{2} a_{2}\right)
$$

and

$G\left(a_{1}, a_{2}\right)=\left(L_{1}\left(-x_{12} \theta_{y_{1}} L_{2} g_{2}\left(a_{1}, a_{2}\right)+g_{1}\left(a_{1}, a_{2}\right)\right), L_{2} g_{2}\left(a_{1}, a_{2}\right)\right)$.

Here, $L *: P C_{T} \times P C_{T} \rightarrow P C_{T} \times P C_{T}$ is linear and relatively compact and $G: P C_{T} \times P C_{T} \rightarrow P C_{T} \times P C_{T}$ is quasi-equicontinuous and relatively compact and meets the condition $G=\circ\left(\left\|\left(a_{1}, a_{2}\right)\right\| P C_{T}\right)$ when $\left\|\left(a_{1}, a_{2}\right)\right\| P C_{T} \rightarrow o$.

Regard $\xi$ in (45) as a real value parameter. As long as $\xi \in R$, any nontrivial periodic solution $\left(a_{1} a_{2}\right) \neq(0,0)$ of (45) on $P C_{T} \times P C_{T}$ can generate a periodic solution $(\beta, \lambda)=$ $\left(a_{1}+\theta_{y_{1}}, a_{2}\right)$ in the collaboration and coexistence model, where $\bar{y}_{2}=\xi$. Hence, the issue of whether the periodic solution of Model (2) exists reduces to the issue of bifurcation of (45). Applying local bifurcation theory to (45), we see that bifurcation occurs only when the linear equation

$$
\left(\delta_{1}, \delta_{2}\right)=\xi L^{*}\left(\delta_{1}, \delta_{2}\right),\left(\delta_{1}, \delta_{2}\right) \neq(0,0), \quad \xi \in R
$$

has a nontrivial solution.

Assume that if $\xi \in R,\left(\delta_{1}, \delta_{2}\right) \in P C_{T} \times P C_{T}$ is a $T$-periodic solution of (48). By the definition of $L^{*},\left(\delta_{1}, \delta_{2}\right)$ is the solution to the following equation

$$
\begin{aligned}
\dot{\delta}_{1}(t) & =\left(y_{1}-2 x_{11} \theta_{y_{1}}\right) \delta_{1}-x_{12} \theta_{y_{1}} \delta_{2}, \quad t \neq \tau_{k}, k \in N, \\
\dot{\delta}_{2}(t) & =\left(p_{2}+\xi-x_{21} \theta_{y_{1}}\right) \delta_{2}, \quad t \neq \tau_{k}, k \in N, \\
\delta_{1}\left(\tau_{k}^{+}\right) & =\left(1+h_{k}\right) \delta_{1}\left(\tau_{k}\right), \quad t=\tau_{k}, k \in N, \\
\delta_{2}\left(\tau_{k}^{+}\right) & =\left(1+g_{k}\right) \delta_{2}\left(\tau_{k}\right), \quad t=\tau_{k}, k \in N,
\end{aligned}
$$

and vice versa. Based on Hypothesis 3(i), if $\xi=\xi_{*}$, where

$$
\xi_{*}=\frac{1}{T} \ln \left[\prod_{k=1}^{q} \frac{1}{1+g_{k}}\right]+\overline{x_{21} \theta_{y_{1}}},
$$

(49) indicates that (45) has the only nontrivial $T$-periodic solution on $P C_{T} \times P C_{T}$. In view of Hypothesis 3(ii), (41) has the only $T$-periodic solution on $P C_{T} \times P C_{T}$ and bifurcation occurs only under the simple eigenvalue $\xi_{*}$. Then, bifurcation of the sectionally continuous periodic solution of (45) can be obtained. Apart from $\left(\xi_{*} ; 0,0\right)$, all the other solutions are nontrivial.

The expanded form of $\left(\xi ; a_{1}, a_{2}\right)$ in the neighbourhood of $\left(\xi_{*} ; 0,0\right)$ suggests that the periodic solution of (45) is equivalent to the positive periodic solution of Model (2). In fact, when $\varepsilon$ is sufficiently small and $a_{i j} \in P T_{T}$, then

$$
\xi=\xi_{*}+\xi_{1} \varepsilon+\cdots, \quad a_{i}=a_{i 1} \varepsilon+a_{i 2} \varepsilon^{2}+\cdots, \quad i=1,2 .
$$

Upon substituting the above equation into (41), and if the coefficients $\varepsilon$ and $\varepsilon^{2}$ on both ends are the same, then

$$
\begin{aligned}
\dot{a}_{11}= & \left(y_{1}-2 x_{11} \theta_{y_{1}}\right) a_{11}-x_{12} \theta_{y_{1}} a_{21}, \quad t \neq \tau_{k}, k \in N, \\
\dot{a}_{21}= & \left(p_{2}+\xi_{*}-x_{21} \theta_{y_{1}}\right) a_{21}, \quad t \neq \tau_{k}, k \in N, \\
a_{11}\left(\tau_{k}^{+}\right)= & \left(1+h_{k}\right) a_{11}\left(\tau_{k}\right), \quad t=\tau_{k}, k \in N, \\
a_{21}\left(\tau_{k}^{+}\right)= & \left(1+g_{k}\right) a_{22}\left(\tau_{k}\right), \quad t=\tau_{k}, k \in N, \\
\dot{a}_{12}= & \left(y_{1}-2 x_{11} \theta_{y_{1}}\right) a_{12}-x_{12} \theta_{y_{1}} a_{22}-x_{11} a_{11}^{2} \\
& -x_{12} a_{11} a_{21}, \quad t \neq \tau_{k}, k \in N, \\
\dot{a}_{22}= & \left(p_{2}+\xi_{*}-x_{21} \theta_{y_{1}}\right) a_{22}+\xi_{1} a_{21}-x_{21} a_{11} a_{21} \\
& -x_{22} a_{21}^{2}, \quad t \neq \tau_{k}, k \in N, \\
a_{12}\left(\tau_{k}^{+}\right)= & \left(1+h_{k}\right) a_{12}\left(\tau_{k}\right), \quad t=\tau_{k}, k \in N, \\
a_{22}\left(\tau_{k}^{+}\right)= & \left(1+g_{k}\right) a_{22}\left(\tau_{k}\right), \quad t=\tau_{k}, k \in N .
\end{aligned}
$$

Hence, $\left(a_{11}, a_{21}\right) \in P C_{T} \times P C_{T}$ is the solution of (48). A special solution meeting the condition of $a_{21}(0)=1$ is

$$
\begin{aligned}
& a_{21}(t)=\prod_{0 \leq \tau_{k}<t}\left(1+g_{k}\right) \exp \int_{0}^{t}\left(p_{2}+\xi_{*}-x_{21} \theta_{y_{1}}\right) \mathrm{d} s>0, \\
& a_{11}(t)=-\int_{0}^{t} G(t, s) x_{12} \theta_{y_{1}} a_{21}(s) \mathrm{d} s .
\end{aligned}
$$

Hence, in terms of $t, a_{11}(t)<0$ is valid. Applying Hypothesis 2 to the second equation in (53), we have $\xi=\overline{x_{21} a_{11}+x_{22} a_{21}}$.

If $\xi_{1}>0$, then there exists a bifurcation $\gamma^{+}=\left\{\left(\xi ; a_{1}, a_{2}\right)\right\}$ $\subseteq R \times P C_{T} \times P C_{T}$, where $\xi>\xi_{*}$, and as long as $t>0$, then $a_{1}(t)<0$ and $a_{2}(t)>0$. When $\xi$ becomes sufficiently close to $\xi_{*}$, it is equivalent to the positive periodic solution of Model (2). Therefore, there exists a $c_{0}>0$ that is sufficiently small to ensure that the bifurcation $C^{+}=\{(\xi ; \beta, \lambda) \in R \times$ $\left.P C_{T} \times P C_{T}\right\}$ can be solved and meets the condition, such that when $\xi_{*}<\xi<\xi_{*}+c_{0}$, if $t>0$, then $\beta(t)<\theta_{y_{1}}$ and $\lambda(t)>0$. Since $\xi_{*}<\xi<\xi_{*}+c_{0}$ is equivalent to $\xi_{*}<\bar{y}_{2}<\xi_{*}+c_{0}$, Hypothesis $5(\mathrm{i})$ is valid. If $\xi_{1}<0$, it is valid similarly that there exists a constant $d_{0}>0$ that is sufficiently small to ensure that the solution $(\beta, \lambda) \in P C_{T} \times P C_{T}$ of Model (2) meets the condition such that when $\xi_{*}-d_{0}<\bar{y}_{2}<\xi_{*}$, if $t>$ 0 , then $\beta(t)<\theta_{y_{1}}$ and $\lambda(t)>0$. The proof is complete.

From Hypothesis 5 , we note that $\xi_{1}$ is a measure of the probability that the two personnel groups coexist. $\xi_{*}$ is a value of the positive periodic solution bifurcated from the semitrivial periodic solution $\left(\theta_{y_{1}}, 0\right)$. If $\xi_{1}>0$, then $\bar{y}_{2}>\xi_{*}$. From Hypothesis 1, we see that the unsteady manifold of the semitrivial positive periodic solution $\left(\theta_{y_{1}}, 0\right)$ increases by one dimension. According to the steadiness exchange principle, when $\xi_{1}>0$, the periodic solution $\left(\bar{y}_{2} ; \beta, \lambda\right)$ exhibits linear asymptotic steadiness. If $\xi_{1}<0$, then the bifurcated periodic solution is unsteady. 


\section{Application Examples}

During the implementation process of complex mega infrastructure projects, many factors, both internal and external, can exert an influence. The engineering personnel groups are not isolated from one another but coexist in a complex engineering system. Once the risk of infection occurs, a chain reaction will be triggered. Therefore, it is necessary to balance the structure of the personnel groups and formulate targeted strategic precautions. The periodic collaboration and coexistence model with the oscillation effect introduced in this study obtains three types of components, namely, a nonnegative periodic solution, a trivial periodic solution $(0,0)$, and semitrivial periodic solutions $\left(\theta_{y_{1}}, 0\right)$ and $\left(0, \theta_{y_{2}}\right)$. Based on the nontrivial $T$-periodic solution bifurcated from the semitrivial periodic solution, the conditions necessary to maintain stability of the trivial periodic solution and the nontrivial periodic solution are obtained. In addition, the conditions obtained suggest that all the trivial periodic solutions and nontrivial periodic solutions are linearly unsteady. This indirectly indicates that personnel groups in the complex major engineering systems discussed in Model (2) can coexist sustainably. The conditions of Hypothesis 2 imply that oscillating periodic collaborative management can prevent one engineering personnel group from being fully assimilated or disappearing after the risk of infection occurs, guaranteeing sustainable production of the major engineering project.

To prove that the internal positive periodic solution bifurcated from the semitrivial periodic solution is stable and steady, we present the following examples and numerical simulation results.

We can use Model (2) to describe periodic collaborative management of two coexisting personnel groups within a complex major engineering system. Since the risk of infection occurs on a discontinuous basis, Model (2) is reasonable. For the convenience of discussion, we assume that an engineering personnel group in any period (such as 1 year) is managed for a finite time period independently. In the other case, two coexisting engineering personnel groups are managed collaboratively during the same period. We can express the oscillation effect as follows:

$$
\begin{aligned}
\dot{\beta}(t) & =\beta(t)\left(\eta+\sin 2 \pi t-x_{11} \beta(t)-x_{12} \lambda(t)\right), \quad t \neq \tau_{k}, k \in Z^{+}, \\
\lambda(t) & =\lambda(t)\left(\xi+\cos 2 \pi t-x_{21} \beta(t)-x_{22} \lambda(t)\right), \quad t \neq \tau_{k}, k \in Z^{+}, \\
\beta\left(\tau_{k}^{+}\right) & =\left(1-p_{1}\right) \beta\left(\tau_{k}\right), \quad t=\tau_{k}, \quad \tau_{k}=\frac{1}{2}(2 k-1), \quad k \in Z^{+}, \\
\lambda\left(\tau_{k}^{+}\right) & =\left(1-p_{2}\right) \lambda\left(\tau_{k}\right), \quad t=\tau_{k}, \quad \tau_{k}=\frac{1}{2}(2 k-1), \quad k \in Z^{+},
\end{aligned}
$$

where $\beta(t)$ and $\lambda(t)$ stand for the density of the two coexisting engineering personnel groups at a certain time point $\eta, \xi$ and $x_{i j}(i, j=1,2)$ are positive constants, $T=1$, and $0 \leq p_{1}<1,0 \leq p_{2}<1$ represent the degrees of collaboration between the two engineering personnel groups. The two components of interest in this research are both

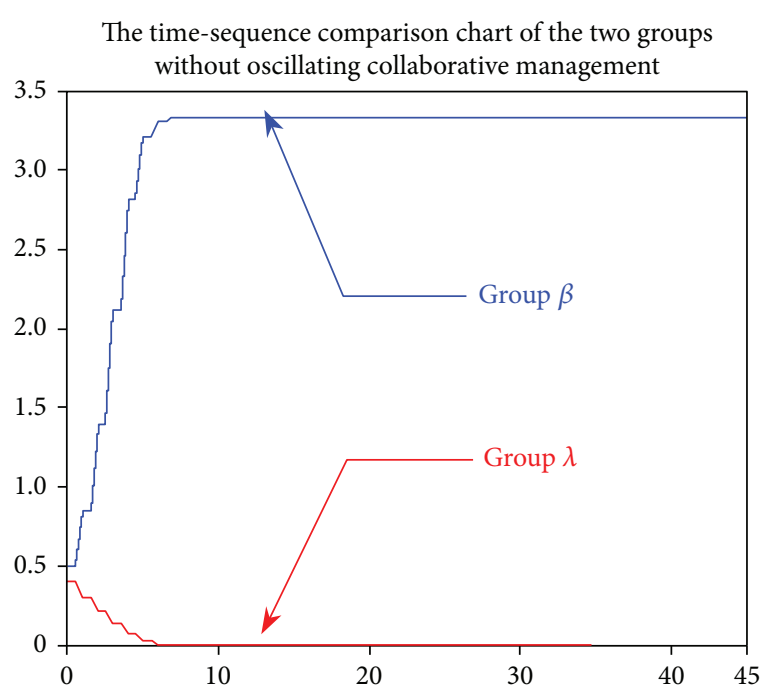

FIgURE 2: The time-sequence comparison chart of the two groups without oscillating collaborative management.

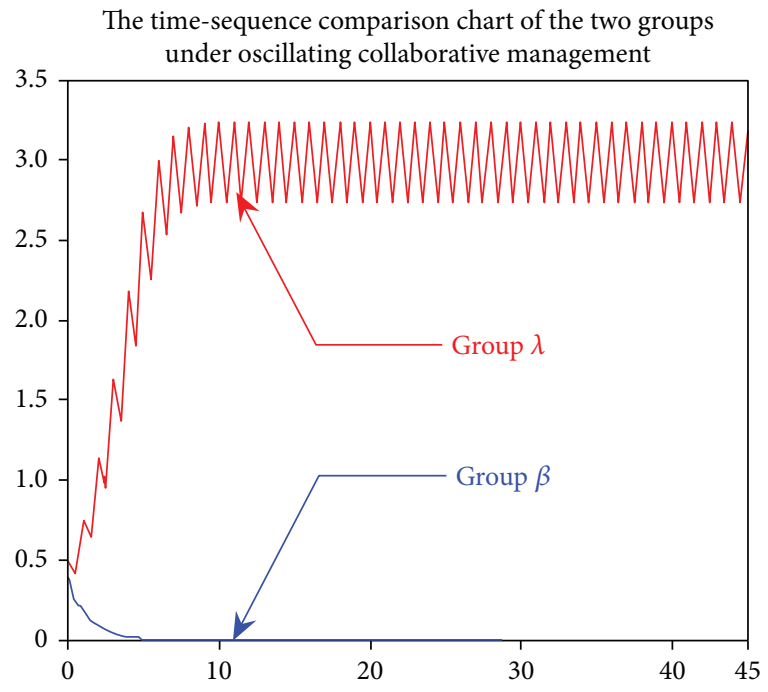

FIgURE 3: The time-sequence comparison chart of the two groups under oscillating collaborative management.

nonnegative solutions. The two engineering personnel groups can coexist.

Assume that $\eta=1, \xi=1, x_{11}=0.4, x_{12}=0.2, x_{21}=0.5$, $x_{22}=0.3$, and $\left(\beta\left(0^{+}\right), \lambda\left(0^{+}\right)\right)=(0.4,0.5)$. Set $p_{1}$ and $p_{2}$ as control variables. System (55) has a semitrivial periodic solution with asymptotic stability when there is no management collaboration $\left(p_{1}=p_{2}=0\right)$.

Figure 2 presents the time-sequence comparison chart of the two groups in Model (55) under the condition of nonoscillating collaborative management. Figure 3 shows the timesequence comparison chart of the two groups in Model (55) under the condition of oscillating collaborative management. As observed from Figures 2 and 3, $\beta$ is the dominant group. If periodic collaborative management is pursued, that 
is, $p_{1}=0.3$ and $p_{2}=0.15$, then the conditions of Hypothesis $2\left(\eta>\ln \left(1 / 1-p_{1}\right)+\left(x_{12} / x_{22}\right)\left(1-\ln \left(1 / 1-p_{2}\right)\right), \quad \xi>\right.$ $\left.\ln \left(1 / 1-p_{2}\right)+\left(x_{21} / x_{11}\right)\left(\eta-\ln \left(1 / 1-p_{2}\right)\right)\right)$ and the conditions of Hypothesis 5 are valid. The two engineering personnel groups coexist in a steady periodic solution. Under the condition and measure of the probability that the two personnel groups coexist, oscillating collaborative management can stop group $\lambda$ from being assimilated or disappearing. In fact, when there is no oscillating collaboration and $\beta$ is the dominant group, and if $p_{2}$ is relatively small, the influence of $\beta$ will decline with increasing $p_{1}$. When the latter continues to increase to a certain extent, the two engineering personnel groups can coexist in a steady periodic solution, fulfilling the management goal.

If $p_{1}$ keeps increasing and when $\eta<\ln \left(1 / 1-p_{1}\right)+\left(x_{12} /\right.$ $\left.x_{22}\right)\left(1-\ln \left(1 / 1-p_{2}\right)\right)$, group $\beta$, as stated in Hypothesis 1(iii), tends to be assimilated or disappear, while group $\lambda$ will periodically oscillate. If the oscillating collaboration value is large, excessive management might lead to the assimilation or disappearance of both groups. Therefore, to guarantee the coexistence of the two kinds of engineering personnel groups, determining an appropriate collaboration and coexistence management strategy is vital.

\section{Conclusions}

Based on the structural characteristics of major engineering personnel groups under the risk of infection, we consider how to inhibit the risk of infection. Based on the theories of dynamic, life cycle, and oscillation, we develop a periodic collaboration and coexistence management model with the oscillation effect. We perform mathematical analysis to show how periodic oscillating collaborative management prevents the full assimilation or disappearance of one engineering personnel group when the risk of infection is triggered, and how the personnel groups in a complex major engineering system can remain balanced to guarantee the sustainability of project construction. We derive the following findings: (1) When the degree of oscillating collaboration is high, excessive management might result in severe imbalance of the personnel structure and impede the complex major engineering project from progressing normally. (2) When infection breaks out, the dominant group will be weakened as collaborative management of the group increases. In addition, when the degree of collaboration reaches a certain degree, the two engineering personnel groups can coexist over a steady period. (3) When two engineering personnel groups exist in a steady period, collaborative management under the oscillating model can prevent the engineering personnel groups from being assimilated or disappearing even under the risk of infection.

In summary, we do not only reveal the mechanism of group collaboration in a complex major engineering system under the risk of infection and enriches the major engineering collaborative management theory but also build a periodic collaboration and coexistence management model with the oscillation effect. The model can provide references for the collaborative management of major engineering systems under the risk of infection. More importantly, it can prevent and curb the occurrence of large-scale infection risk, thus safeguarding the structural stability of the complex major engineering system. Taken together, our research findings complement and supplement the pertinent theories and provide useful references for improving collaborative management in complex mega infrastructure projects. However, this study is in the theoretical research stage of mechanism revelation and model establishment. We will continue to enrich this theory in future research and try to do some research on simulation and experiment.

\section{Data Availability}

No data were used to support this study.

\section{Conflicts of Interest}

The authors declare that there is no conflict of interests regarding the publication of this paper.

\section{Acknowledgments}

This work was supported by the National Natural Science Foundation of China (Grant nos. 71390522 and 71601058), the Natural Science Foundation of Heilongjiang of China (Grant number QC2017084), and Heilongiiang Postdoctoral Foundation of China.

\section{References}

[1] S. X. Zeng, H. Y. Ma, H. Lin, R. C. Zeng, and V. W. Y. Tam, "Social responsibility of major infrastructure projects in China," International Journal of Project Management, vol. 33, no. 3, pp. 537-548, 2015.

[2] C. E. Maldonado, The Complexification of Engineering, John Wiley \& Sons, Inc., 2012.

[3] L. Varga, T. Grubic, P. Greening, S. Varga, F. Camci, and T. Dolan, "Characterizing conversion points and complex infrastructure systems: creating a system representation for agent-based modeling," Complexity, vol. 19, no. 6, 43 pages, 2014.

[4] P. T. I. Lam, "A sectoral review of risks associated with major infrastructure projects," International Journal of Project Management, vol. 17, no. 2, pp. 77-87, 1999.

[5] F. Solis, J. V. Sinfield, and D. M. Abraham, "Hybrid approach to the study of inter-organization high performance teams," Journal of Construction Engineering and Management, vol. 139, no. 4, pp. 379-392, 2013.

[6] L. Calamel, C. Defélix, T. Picq, and D. Retour, "Inter-organisational projects in French innovation clusters: the construction of collaboration," International Journal of Project Management, vol. 30, no. 1, pp. 48-59, 2012.

[7] J. M. Ottino, "Engineering complex systems," Nature, vol. 427, no. 6973 , pp. 399-399, 2004.

[8] B. Sauser and J. Boardman, "Taking hold of system of systems management," Engineering Management Journal, vol. 20, no. 4, pp. 3-8, 2008.

[9] R. Abbott, "Complex systems engineering: putting complex systems to work," Complexity, vol. 13, no. 2, 11 pages, 2007.

[10] J. Du and M. El-Gafy, "Virtual organizational imitation for construction enterprises: agent-based simulation framework for exploring human and organizational implications in 
construction management," Journal of Computing in Civil Engineering, vol. 26, no. 3, pp. 282-297, 2012.

[11] G. Hofstede, G. H. Hofstede, and G. J. Hofstede, Cultures and Organizations, McGraw-Hill, 1997.

[12] T. Varvel, S. G. Adams, S. J. Pridie, and B. C. Ruiz Ulloa, "Team effectiveness and individual Myers-Briggs personality dimensions," Journal of Management in Engineering, vol. 20, no. 4, pp. 141-146, 2004.

[13] P. Mitropoulos and B. Memarian, "Team processes and safety of workers: cognitive, affective, and behavioral processes of construction crews," Journal of Construction Engineering and Management, vol. 138, no. 10, pp. 1181-1191, 2012.

[14] T. Braun, A. I. Ferreira, and J. Sydow, "Citizenship behavior and effectiveness in temporary organizations," International Journal of Project Management, vol. 31, no. 6, pp. 862-876, 2013.

[15] R. Dwivedula and C. N. Bredillet, "The relationship between organizational and professional commitment in the case of project workers: implications for project management," Project Management Journal, vol. 41, no. 4, pp. 79-88, 2010.

[16] S. Comu, J. Iorio, J. E. Taylor, and C. S. Dossick, "Quantifying the impact of facilitation on transactive memory system formation in global virtual project networks," Journal of Construction Engineering and Management, vol. 139, no. 3, pp. 294-303, 2013.

[17] H. Thornton, An Enquiry into the Nature and Effects of the Paper Credit of Great Britain, Routledge, 2017.

[18] N. M. Almeida, V. Sousa, L. A. Dias, and F. Branco, "Engineering risk management in performance-based building environments," Journal of Civil Engineering \& Management, vol. 21, no. 2, pp. 218-230, 2015.

[19] J. A. Conger and R. N. Kanungo, "The empowerment process: integrating theory and practice," Academy of Management Review, vol. 13, no. 3, pp. 471-482, 1988.

[20] G. Katalin, T. Meggyes, and E. Fenyvesi, Engineering Tools for Environmental Risk Management, CRC Press, 2015.

[21] T. C. E. Cheng and Q. Ding, "Scheduling start time dependent tasks with deadlines and identical initial processing times on a single machine," Computers \& Operations Research, vol. 30, no. 1, pp. 51-62, 2003.

[22] M. Bresnen and N. Marshall, "Building partnerships: case studies of clientcontractor collaboration in the UK construction industry," Construction Management and Economics, vol. 18, no. 7, pp. 819-832, 2000.

[23] U. Horst, "Stochastic cascades, credit contagion, and large portfolio losses," Journal of Economic Behavior \& Organization, vol. 63, no. 1, pp. 25-54, 2007.

[24] M. Bosch-Rekveldt, Y. Jongkind, H. Mooi, H. Bakker, and A. Verbraeck, "Grasping project complexity in large engineering projects: the TOE (Technical, Organizational and Environmental) framework," International Journal of Project Management, vol. 29, no. 6, pp. 728-739, 2011.

[25] M. J. Stern and K. J. Coleman, "The multidimensionality of trust: applications in collaborative natural resource management," Society \& Natural Resources, vol. 28, no. 2, pp. 117132, 2015.

[26] J. N. Warfield, "Twenty laws of complexity: science applicable in organizations," Systems Research and Behavioral Science, vol. 16, no. 1, pp. 3-40, 1999.

[27] D. N. Antoniadis, F. T. Edum-Fotwe, and A. Thorpe, "Socioorgano complexity and project performance," International
Journal of Project Management, vol. 29, no. 7, pp. 808-816, 2011.

[28] R. R. Senescu, G. Aranda-Mena, and J. R. Haymaker, "Relationships between project complexity and communication," Journal of Management in Engineering, vol. 29, no. 2, pp. 183-197, 2013.

[29] B. Pauget and A. Wald, "Relational competence in complex temporary organizations: the case of a French hospital construction project network," International Journal of Project Management, vol. 31, no. 2, pp. 200-211, 2013.

[30] N. Zhao and S. An, "Collaborative management of complex major construction projects: anylogic-based simulation modelling," Discrete Dynamics in Nature and Society, vol. 2016, Article ID 6195673, 8 pages, 2016.

[31] T. J. Wheeler, “Analysis, modeling, emergence \& integration in complex systems: a modeling and integration framework \& system biology," Complexity, vol. 13, no. 1, 75 pages, 2007. 


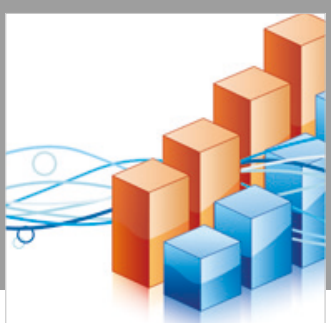

Advances in

Operations Research

\section{-n-m}



Journal of

Applied Mathematics
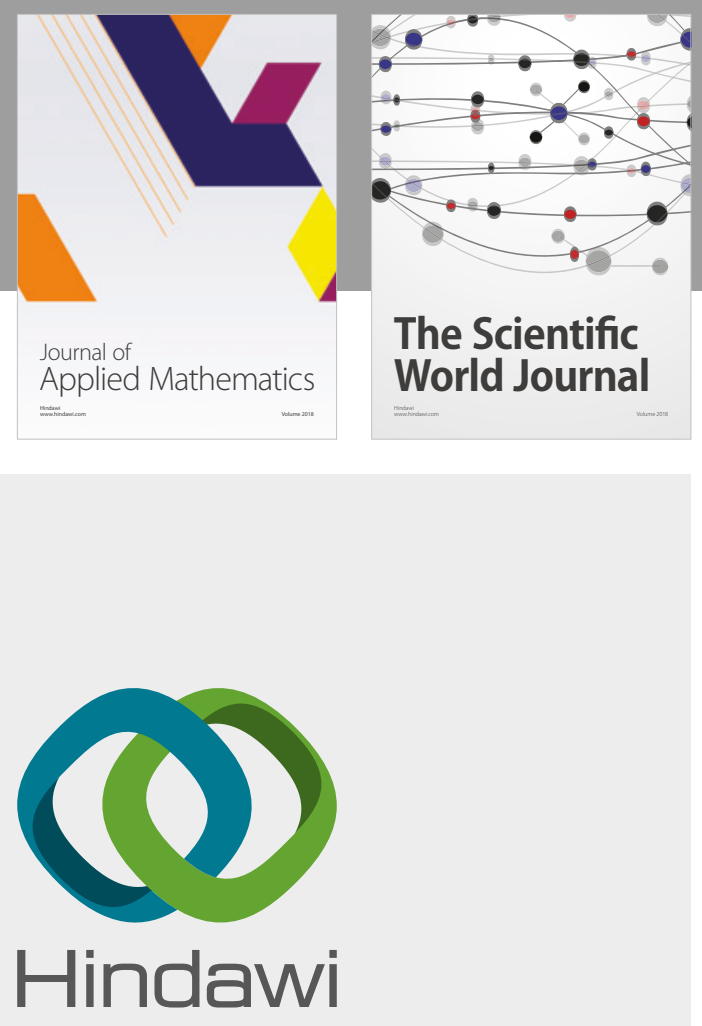

Submit your manuscripts at

www.hindawi.com

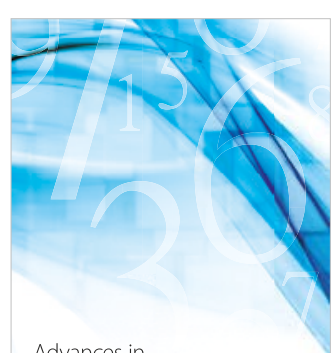

Advances in
Numerical Analysis
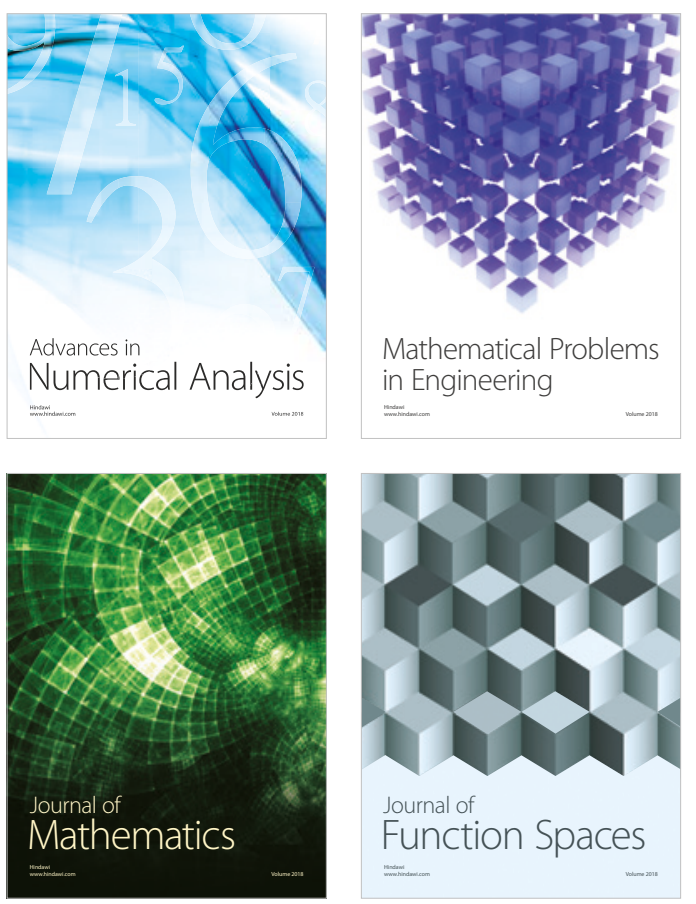

Mathematical Problems in Engineering

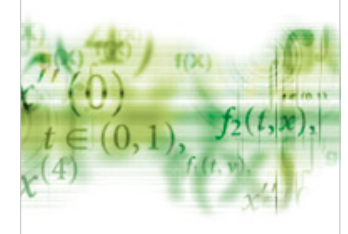

International Journal of

Differential Equations

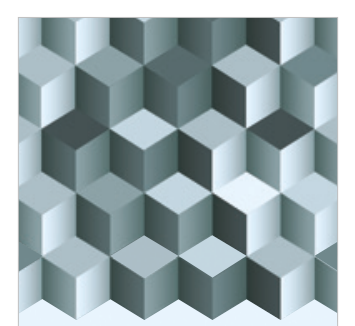

Journal of

Function Spaces
The Scientific

World Journal

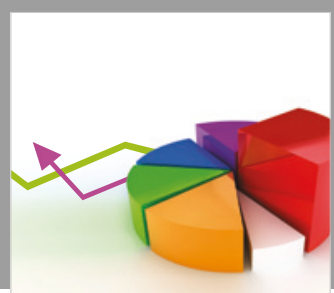

Journal of

Probability and Statistics
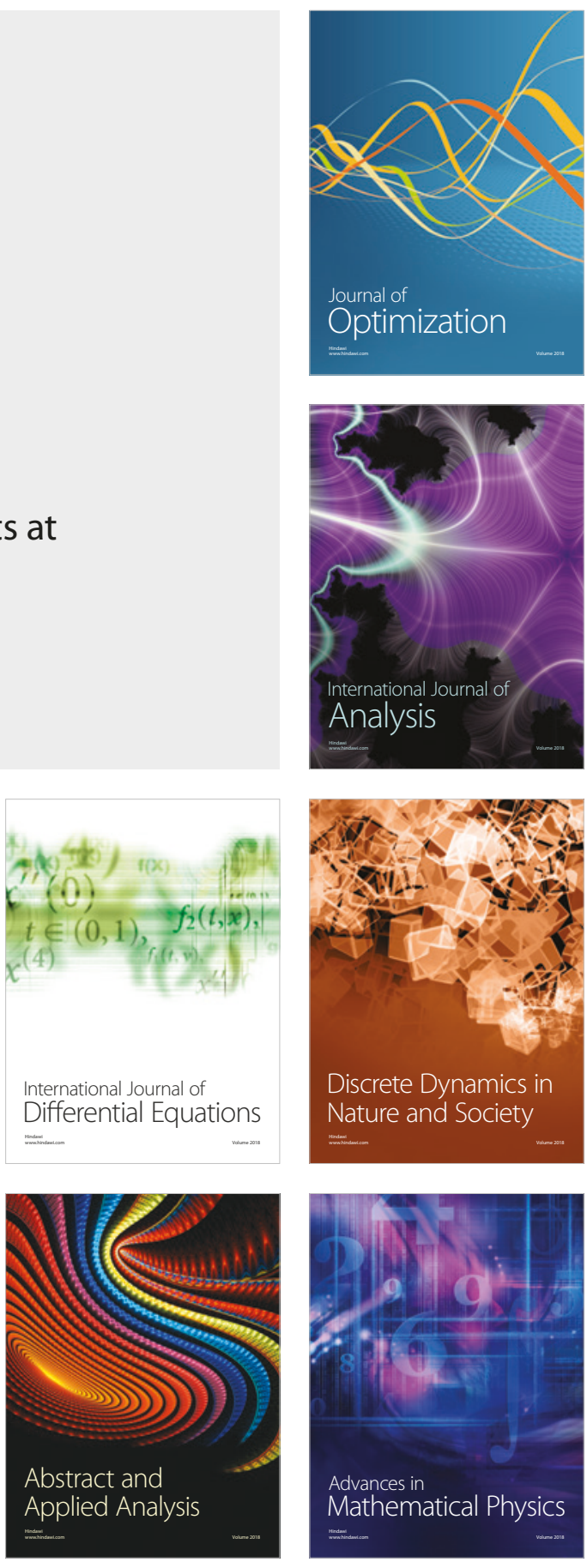